\title{
DIFICULTADES DEL PROFESORADO EN FORMACIÓN AL APLICAR LA EVALUACIÓN FORMATIVA Y COMPARTIDA EN SUS PRIMERAS EXPERIENCIAS DOCENTES
}

Pre-service teachers' difficulties in applying formative and shared assessment in their first teaching experiences

Dificuldades dos professores em formaçãona aplicação da avaliação formativa e partilhada nas suas primeiras experiências docentes

\section{Eloísa Lorente-Catalán}

INEFC-UdL, España. Teléfono: +34 973272022. Correo electrónico: elorente@inefc.es

\section{Resumen}

A pesar de los progresos realizados en los últimos 20 años, no podemos afirmar que la evaluación formativa y compartida sea una práctica regular, integrada, extendida y productiva educativamente en el ámbito de la Educación Física. Algunas investigaciones apuntan a que el hecho de experimentar en la formación inicial este tipo de evaluación da confianza a los futuros profesionales para aplicarla en su futura práctica profesional. Sin embargo, la transferencia no es tan sencilla y directa. En muchas ocasiones, las dificultades encontradas al aplicar dichas prácticas en sus primeras experiencias docentes son motivo de frustración y abandono de las mismas. Por ello, será interesante identificar qué dificultades encuentran los profesores aún en formación en esas primeras prácticas docentes cuando aún están vinculados directamente a la universidad (prácticas de máster) para así poder orientar mejor la formación inicial de dicho profesorado.

Palabras clave: Evaluación formativa, evaluación compartida, formación inicial del profesorado, transferencia, educación física

\section{Abstract}

Despite the progress made in the last 20 years, we are not able to state that formative and shared assessment is a regular, integrated, extended and educationally productive 
practice in the field of Physical Education. Some researches related to this subject suggest that the fact of experiencing this type of assessment in Teacher Education gives confidence to future professionals to apply it in their future professional practice. However, the transfer is not so simple and direct. On many occasions, the difficulties encountered in applying these practices in their first teaching experiences are cause of frustration and abandonment of them. Therefore, it is interesting to identify what difficulties pre-service teachers still find in these early teaching practices when they are still directly linked to the university (school placement) in order to better guide the initial training of these teachers.

Keywords: Formative assessment, shared assessment, teacher education, transference, physical education

\section{Resumo}

Apesar dos avanços alcançados nos últimos 20 anos, não podemos afirmar que a avaliação formativa e partilhada seja uma prática regular, integrada, abrangente e produtiva educativamente na área da educação física. Algumas investigações indicam que o facto de experimentar este tipo de avaliação na formação inicial dá confiança aos futuros profissionais para aplicá-lo na sua prática profissional futura. No entanto, a transferência não é assim tão simples e direta. De uma forma geral, as dificuldades encontradas na aplicação destas práticas nas suas primeiras experiências de ensino são motivo de frustração e abandono das mesmas. Desse modo, será interessante identificar quais as dificuldades encontradas pelos professores em formação, nessas primeiras práticas de ensino, quando estes ainda estão em formação na universidade (estágio do mestrado), para deste modo, puder orientar melhor a formação inicial desses professores.

Palavras chave: Avaliação formativa, avaliação partilhada, formação inicial de professores, transferência, educação física

\section{Introducción}

El profesorado de Educación Física $(\mathrm{EF})$ tradicionalmente ha experimentado dificultades para evaluar el aprendizaje del alumnado (Hay and Penney, 2009; LópezPastor, Kirk, Lorente-Catalán, McPhail y McDonald, 2013). Posiblemente esta sea una 
de las razones por las que la evaluación en esta área del currículum todavía esté, en muchos casos, vinculada al uso de test físicos cuyo objetivo es evaluar el rendimiento físico del alumnado (López-Pastor et al, 2013).

Aunque la amplia revisión internacional de investigaciones sobre evaluación en EF realizada por López et al. (2013) muestra un importante progreso en esta área, su trabajo también muestra que estas prácticas de evaluación más centradas en el aprendizaje están lejos de ser regulares, integradas, extendidas y productivas educativamente (LorenteCatalán y Kirk, 2016). Por ello, uno de los retos para el profesorado de EF es pasar de modelos tradicionales de evaluación-calificación (test físicos y motores) a sistemas y estrategias dirigidos a mejorar el aprendizaje como la evaluación formativa $y$ compartida (López-Pastor et al., 2013).

\section{La experimentación de la evaluación formativa y compartida en la formación} inicial del profesorado y su transferencia a las primeras experiencias profesionales

Si queremos afrontar el reto anteriormente mencionado es preciso que en la formación inicial del profesorado (FIP) se utilice también este tipo de evaluación, no solo por los beneficios que genera sobre el aprendizaje de competencias docentes sino porque hay evidencias de que experimentar estas estrategias durante la misma influye sobre la evaluación que aplican los maestros en su práctica docente (Hamodi et al., 2017; Molina y López-Pastor, 2019). No obstante, no es una cuestión sencilla y directa, ni exenta de dificultades.

Por otro lado, el alumnado que ha vivenciado en su FIP este tipo de evaluación y después la ha aplicado en las primeras experiencias en el prácticum del grado o en el máster, se suele mostrar dispuesto a seguir utilizándolas en su futura práctica profesional (Lorente-Catalán y Kirk, 2016; Lorente-Catalán, López-Pastor y Kirk, 2018). Sin embargo, también es cierto que no siempre estas intenciones se materializan en su práctica docente futura. Cuando el profesorado encuentra dificultades la primera vez que aplica esta evaluación formativa y compartida tiende a abandonar estas prácticas. Por ello, será importante que esas primeras experiencias sean satisfactorias. El análisis de estas primeras prácticas profesionales nos puede ayudar a identificar qué dificultades encuentran al aplicar dichas estrategias, cómo las solventan y si existen 
concepciones erróneas respecto a lo que se ha enseñado en la universidad, lo cual nos proporcionará importantes pistas sobre como reorientar la formación del profesorado.

\section{Principales dificultades encontradas por el profesorado en formación}

Los resultados que se presentan aquí son el fruto de continuos ciclos de investigaciónacción realizados en los últimos cuatro cursos académicos en el contexto de un máster de formación del profesorado de secundaria, bachillerato y ciclos formativos en su especialidad de EF pero posiblemente serían transferibles a otras disciplinas o áreas de conocimiento. Han sido extraídos a partir de cuestionarios elaborados ad hoc, grupos focales $\mathrm{y}$ de entrevistas semiestructuradas realizados al profesorado en formación después de realizar siete semanas de prácticas en los centros educativos. En dichas prácticas diseñan y llevan a cabo como mínimo una unidad didáctica, siendo responsables también de la evaluación y calificación del alumnado.

Entre las dificultades encontradas los estudiantes mencionan la baja experiencia del alumnado de secundaria en el uso de este tipo de evaluación -especialmente la compartida- lo que genera problemas de comprensión sobre el funcionamiento de estos procesos. Durante dichas prácticas el profesorado novel no tiene suficiente tiempo para “entrenar" al alumnado en la autoevaluación o la evaluación entre iguales, por lo que, en muchas ocasiones, se queda en un intento precipitado y mal aplicado. El profesorado en formación culpa al alumnado de falta de sinceridad y de cierta tendencia a no ser objetivos. También describen una resistencia inicial de éstos hacia dichas estrategias. Por otro lado, les resulta difícil gestionar el tiempo que requiere revisar todas las producciones del alumnado, así como el llevar a cabo las tutorías de evaluación para la evaluación compartida, lo cual sumado al estrés que genera una experiencia en un contexto nuevo o poco conocido, lleva, en muchos casos, a la frustración.

Entre los errores cometidos durante la aplicación de la autoevaluación y la evaluación entre iguales cabe destacar: 1) no dejar claro desde el principio lo que el alumnado tiene que evaluarse 2) excesivo número de aspectos a evaluar; 4) instrumentos centrados en la calificación y no en el aprendizaje; 5) utilizar estas estrategias para calificar sin un entrenamiento previo del alumnado; y 6) no ser conscientes de que aprender a autorregularse requiere de un proceso progresivo de toma de decisiones, de un entrenamiento. 


\section{Conclusiones}

Muchas de las dificultades encontradas han sido advertidas a los futuros docentes desde la FIP, se les ha dado consejos y sugerencias para llevar a cabo procesos de evaluación formativa y compartida de calidad, pero la realidad nos muestra que estos consejos no cobran verdadero significado hasta que no los experimentan por sí mismos. De esta manera, los errores cometidos no son un problema sino oportunidades de aprendizaje. Sin embargo, para que el fracaso no les lleve a desestimar el uso de estas prácticas evaluativas en un futuro, se hace necesario un proceso continuo de reflexión de los y las estudiantes con sus tutores y mentores. Durante este período también será de vital importancia la existencia de un discurso coherente entre la escuela y la universidad si queremos que este impacte suficientemente en las teorías y creencias de los futuros docentes. De lo contrario, tenderán a reproducir lo que sus profesores y profesoras hicieron con ellos cuando eran estudiantes (una evaluación sumativa y cuantitativa) en lugar de hacer caso de la formación específica recibida (transmitida y vivenciada) sobre la evaluación formativa y compartida en la universidad.

\section{Referencias}

Hamodi, C., López-Pastor, V. M. y López-Pastor, A. T. (2017). If I experience formative assessment whilst studying at university, will put it into practice later as a teacher? Formative and shared assessment in Initial Teacher Education (ITE). European Journal of Teacher $\quad$ Education, 171-190. https://doi.org/10.1080/02619768.2017.1281909

Hay, P. \& Penney, D. (2009). Proposing conditions for assessment efficacy in physical education. EPER, 5(3) 389-405.

López-Pastor, V., Kirk, D, Lorente-Catalán, E., McPhail, A. \& Mcdonald, D. (2013). Alternative assessment in physical education: a review of international literature. Sport, Education and Society, 18.1, 57-76.

Lorente-Catalán, E. \& Kirk, D. (2016). Student teachers' understanding and application of assessment for learning during a physical education teacher education course. EPER, 22(1) 65-81.

Lorente-Catalán, E., López-Pastor, V.M. y Kirk, D. (2018). La evaluación participativa en la formación inicial del profesorado. Un caso sobre su utilización en las primeras experiencias profesionales. En E. Lorente-Catalán, E. y D. Martos (ed.) (2018) 
Educación Física y Pedagogía crítica. Propuestas para la transformación personal y social (193-209). Edicions de la Universitat de Lleida.

Molina, M y López-Pastor, V. (2019). ¿Evalúo cómo me Evaluaron en la Facultad? Transferencia de la Evaluación Formativa y Compartida Vivida durante la Formación Inicial del Profesorado a la Práctica como Docente. Revista Iberoamericana de Evaluación Educativa, 2019, 12(1), 85-101. https://doi.org/10.15366/riee2019.12.1.005 